\title{
USO DE VANT COMO FERRAMENTA PARA ESTIMATIVA DE ALTURA DE DOSSEL EM MANGUEZAL: UM ESTUDO INVESTIGATIVO EM GUARATIBA, RIO DE JANEIRO, BRASIL
}

\section{Use of RPA as a tool for estimating canopy height in mangrove: an investigative study in Guaratiba, Rio de Janeiro, Brazil}

\author{
Caio de Lima Boa Morte \\ Universidade Federal do Rio de Janeiro \\ caiolboamorte@gmail.com \\ Luiz Felipe Salomon Guaycuru de Carvalho \\ Universidade Federal do Rio de Janeiro \\ luiz_salomon@poli.ufrj.br \\ Rafael Silva de Barros \\ Universidade Federal do Rio de Janeiro \\ rafael.barros@igeo.ufrj.br
}

Artigo enviado para publicação em 31/10/2020 e aceito em 01/12/2020

DOI: $10.12957 /$ tamoios.2020.55745

\begin{abstract}
Resumo
Estimativas para altura de árvores são valores difíceis de se obter se não houver acesso a dados LIDAR. Contudo, VANTs (Veículos Aéreos Não-Tripulados) ou drones podem gerar MDS (Modelos Digitais de Superfície) muito precisos. O objetivo deste estudo é investigar as características altimétricas que podem ser obtidas através do imageamento com VANT, em uma formação de mangue em Guaratiba, Rio de Janeiro - Brasil, subtraindo o MDT (Modelo Digital de Terreno) do MDS gerado. O resultado é um Modelo Digital de Copas (MDC), que pode ser usado como estimativa da altura da vegetação em grande detalhamento. Os resultados apresentam grande precisão planimétrica e altimétrica para o ortomosaico e para o MDS. Considerando erros abaixo de $15 \mathrm{~cm}$ para o DSM (Boa Morte, 2018) e variações abaixo de 75 centímetros para cotas altimétricas do terreno na área de estudos, a estimativa do erro do MDC é menor que 1 metro.
\end{abstract}

Palavras-chave: Drone; MDS; MDC; MDT; Mangue

\begin{abstract}
Estimatives for tree heights are difficult if there is no LIDAR data available. However, UAVs (Unmanned Aerial Vehicle) or drones may generate very accurated DSM (Digital Surface Model). The aim of this study is to investigate altimetric characteristics that can be obtained through UAV imagery of a 3 hectares mangrove formation at Guaratiba, Rio de janeiro - Brasil, by subtracting the DSM by DTM. The result is a Digital Canopy Model (CDM) that can be used as a way to estimate the vegetation height with high detail. Results presented high planimetric and altimetric accuracy for the orthophotomosaic and for the DSM. Since the maximum error was under 15 centimeters for the DSM and the terrain heights variantion were under 75 centimeters at study area, the estimative for the DCM error is lower than 1 meter.
\end{abstract}

Keywords: Drone; DCM; DSM; DTM; Mangrove 


\section{Introdução}

A determinação da altura de árvores, em geral, é uma tarefa pouco exata dadas as dificuldades envolvidas: visualizar e/ou acessar a parte mais alta de uma árvore não é fácil, especialmente dentro de uma floresta. Muitas estimativas dependem da visualização do topo da árvore e usam técnicas visuais e/ou cálculos geométricos. Os resultados acabam apresentando exatidões muito variáveis dependendo das condições, do operador, do método. O uso de LIDAR aumenta em muito a exatidão destas estimativas, mas com seu custo ainda muito elevado, nem sempre se tem acesso a tais dados. Para áreas pequenas (até algumas poucas centenas de hectares) e com pouca variação na altimetria, a utilização de VANT (Veículo Aéreo Não Tripulado) pode trazer uma melhoria nestas estimativas de altura de copas das árvores.

O presente trabalho tem como objetivo elaborar um estudo investigativo acerca das características altimétricas que podem ser obtidas a partir da análise de um MDS produzido por meio de fotografias aéreas de VANT multirotor de uso comercial sobre área com cobertura de mangue na Reserva Estadual de Guaratiba. A área de interesse engloba cerca de 3 hectares e pretende-se obter por resultado a estimativa da altura das copas das árvores que cobrem o solo naquela área a partir da diferença de elevação entre o Modelo Digital de Superfície (MDS) e o Modelo Digital de Terreno (MDT) estimado a partir do MDS, dada a altíssima resolução das imagens obtidas para composição do modelo.

De acordo com Lagomasino et al. (2016), a métrica da altura da vegetação é um dado importante pois ela é um dos dados necessários para o cálculo de estoque de carbono. Através desse cálculo é possível mensurar a quantidade de gases do efeito estufa que podem ser lançados na atmosfera caso determinada vegetação não seja conservada. Um outro fator importante da medida do estoque de carbono é a possibilidade de captação de recursos a partir de medidas REDD+ (Redução de Emissões decorrentes do Desmatamento e da Degradação de florestas), que recompensam financeiramente projetos e instituiçõos com base nos seus resultados na redução da emissão de gases estufa. Além das questões relacionadas a estoque de carbono, de diversas outras aplicações que envolvem o mapeamento da vegetação se beneficiam de uma boa estimativa da sua altura, como por exemplo em Coelho 2019). A escolha de uma área de manguezal como área de estudo se mostra ainda mais relevante já que, como Donato et al.(2011) afirmam, no ritmo atual de degradação os cortes dos mangues podem ser responsáveis por até $10 \%$ das emissões de carbono por desflorestamento globalmente, mesmo que esse ecossistema cubra apenas $3 \%$ da cobertura vegetal mundial.

Como apontado por Coelho (2019) e Boa Morte (2019), o Modelo Digital de Superfície (MDS) resultante do processamento dos dados dos VANTs é uma importante métrica para diferenciação de estruturas vegetais, sendo em alguns casos um dado tão relevante quanto as imagens em infravermelho próximo (NIR), pois ele permite perceber diferenças na vegetação pelas suas diferenças de altura. Uma outra possível análise a ser feita a partir do MDS é a geração de um Modelo Digital de Terreno (MDT), uma vez que através da extração de dados planimétricos e altimétricos de determinados pontos de solo exposto pode-se realizar uma interpolação dos mesmos e, assim, obter-se uma estimativa de como o terreno se desenvolve sob os elementos naturais ou antrópicos. Esta possibilidade deve ser restrita a áreas cuja variação na altimetria possa ser considerada aceitável para o objetivo do estudo.

A utilização de VANTs em projetos ambientais tem se tornado uma prática cada vez mais comum atualmente, desde o monitoramento ambiental até a produção de classificações de vegetação. Tal aumento se dá pela constante evolução tecnológica e 
subsequente barateamento dessa tecnologia. Suas principais vantagens são a alta resolução dos seus produtos, a relativa facilidade e flexibilidade de implementação e seu menor custo se comparado ao uso de imagens orbitais de altíssima resolução ou de fotografias aéreas, além do maior dinamismo e independência na aquisição.

O emprego de VANTs em estudos ambientais pode ser uma grande ferramenta para o aumento do detalhamento nesses estudos, porém ainda existem algumas limitações. Uma delas é a área passível de mapeamento em uma única missão. Enquanto imagens orbitais conseguem abranger grandes áreas em uma só imagem, os VANTs são limitados a pequenas áreas devido a alguns fatores como a baixa duração de suas baterias (em modelos menores e mais baratos), a altura máxima de voo permitido para usuários sem brevê etc. Outro problema comumente encontrado é a necessidade de se utilizar Pontos de Controle no Terreno (em inglês Ground Control Points - GCP) para corrigir erros planimétricos e altimétricos decorrentes da baixa precisão e acurácia cartográfica dos módulos GNSS (Sistemas Globais de Navegação por Satélite) embarcados nesses equipamentos. Os GCPs são alvos físicos georreferenciados com aparelhos GNSS de alta precisão que devem ser distribuídos dentro da área de interesse e são usados como referência durante a fase do processamento dos dados. Uma forma de evitar essa etapa é a utilização de VANTs com módulos GNSS RTK embarcados, que geram produtos com precisão e acurácia cartográfica muito próximos da utilização de GCPs, sem a perda de tempo da distribuição e medição dos alvos físicos pela área de estudo.

\section{Metodologia}

A área de estudo deste trabalho (Figura 1) é uma pequena franja de Mangue próxima à praia de Araçatiba, no Canal da Varzinha, na Reserva Estadual da Guaratiba no município do Rio de Janeiro-RJ, com uma área imageada total de aproximadamente 23 hectares, porém com análises feitas em apenas um recorte de aproximadamente 3 hectares. A área de estudo foi escolhida devido ao seu nível maior de acessibilidade, em vista da necessidade da utilização de GCPs, e pela relevância ecológica do ecossistema.

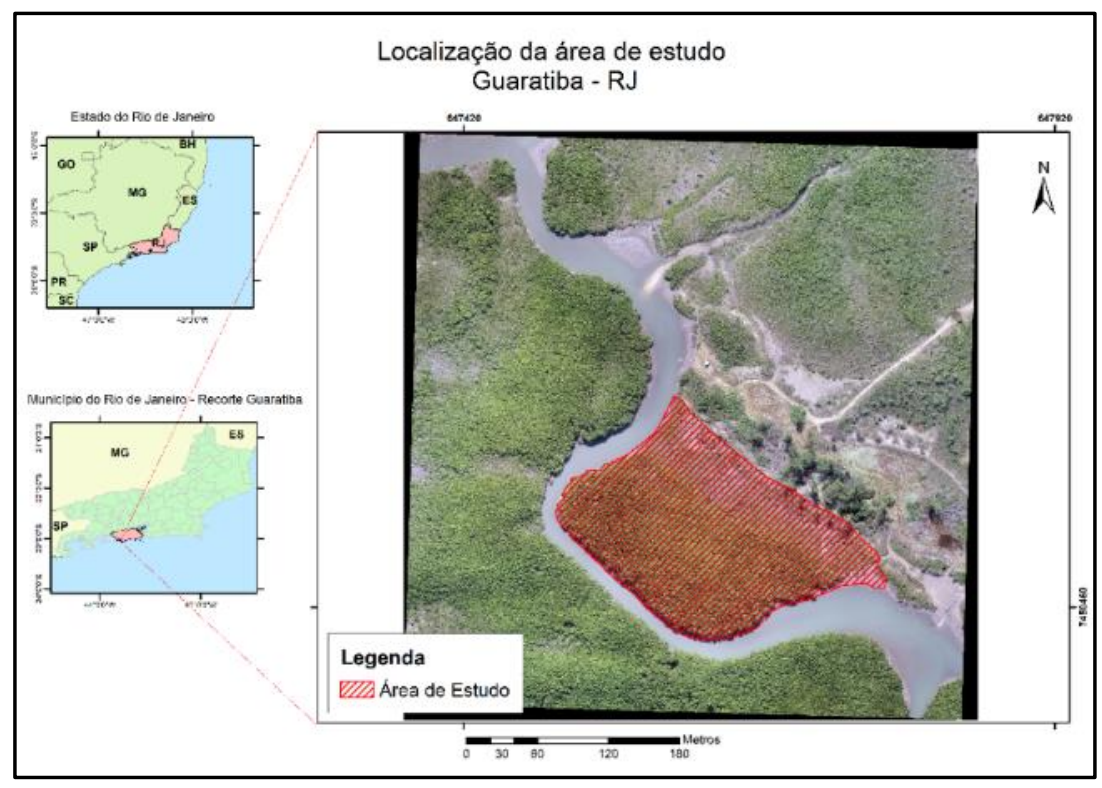

Figura 1: Localização da área de estudo 
Para adquirir as fotografias aéreas foi realizado um trabalho de campo no dia 16 de agosto de 2019, no qual, através de dois voos complementares e subsequentes, foram tomadas 656 fotos entre 11:55hrs e 12:30 horas, a uma altura de 120 metros, com sobreposição de $85 \%$ na longitudinal e $70 \%$ na lateral, gerando um Ortofotomosaico e um Modelo Digital de Superfície, ambos com resoluções de $3,2 \mathrm{~cm}$. Para a correção planimétrica e altimétrica, foram distribuídos 5 GCPs para a correção geométrica e 6 Checkpoints (CPs) para a avaliação da exatidão cartográfica dos produtos. Para se obter as coordenadas dos alvos com alta precisão foram utilizados dois rastreadores GNSS Trimble R6 (Figura 2) no modo Relativo Cinemático, onde um foi utilizado como base fixa, adquirindo coordenadas por mais de $4 \mathrm{hrs}$, e o outro como unidade móvel para adquirir as coordenadas dos

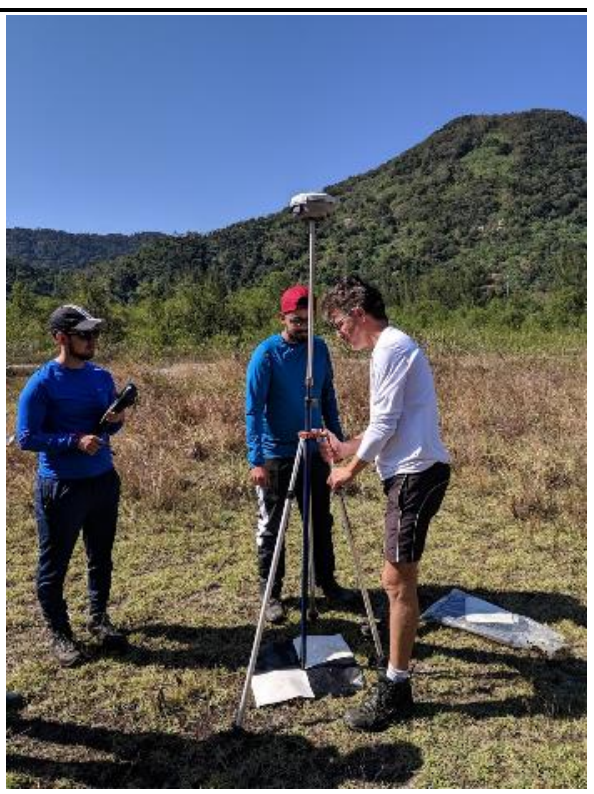

Figura 2: Determinação de coordenadas geográficas de alta precisão com GNSS centros dos alvos. Em gabinete, após 15 dias, os dados adquiridos pelo rastreador na base fixa foram processados com os dados de órbita precisa pelo serviço IBGE-PPP. Em seguida os dados referentes aos demais pontos foram processados em relação aos da base fixa. Por fim, o sensor óptico utilizado foi o RGB embarcado no VANT multirotor Phantom 4 Pro, da fabricante DJI.

Para o processamento dos dados de campo foi utilizado o software de fotogrametria Pix4DMapper e uma workstation com um CPU de 6 núcleos Intel Xeon E5-1650 v4 @ 3.60GHz, 128gb de memória RAM e uma GPU Nvidia Quadro K1200. Para a geração do Ortofotomosaico e do MDS, cada GCP utilizado foi marcado em 10 fotos, sempre com boa identificação dos alvos. A figura 3, a seguir, apresenta a distribuição dos GCPs e CPs na área.

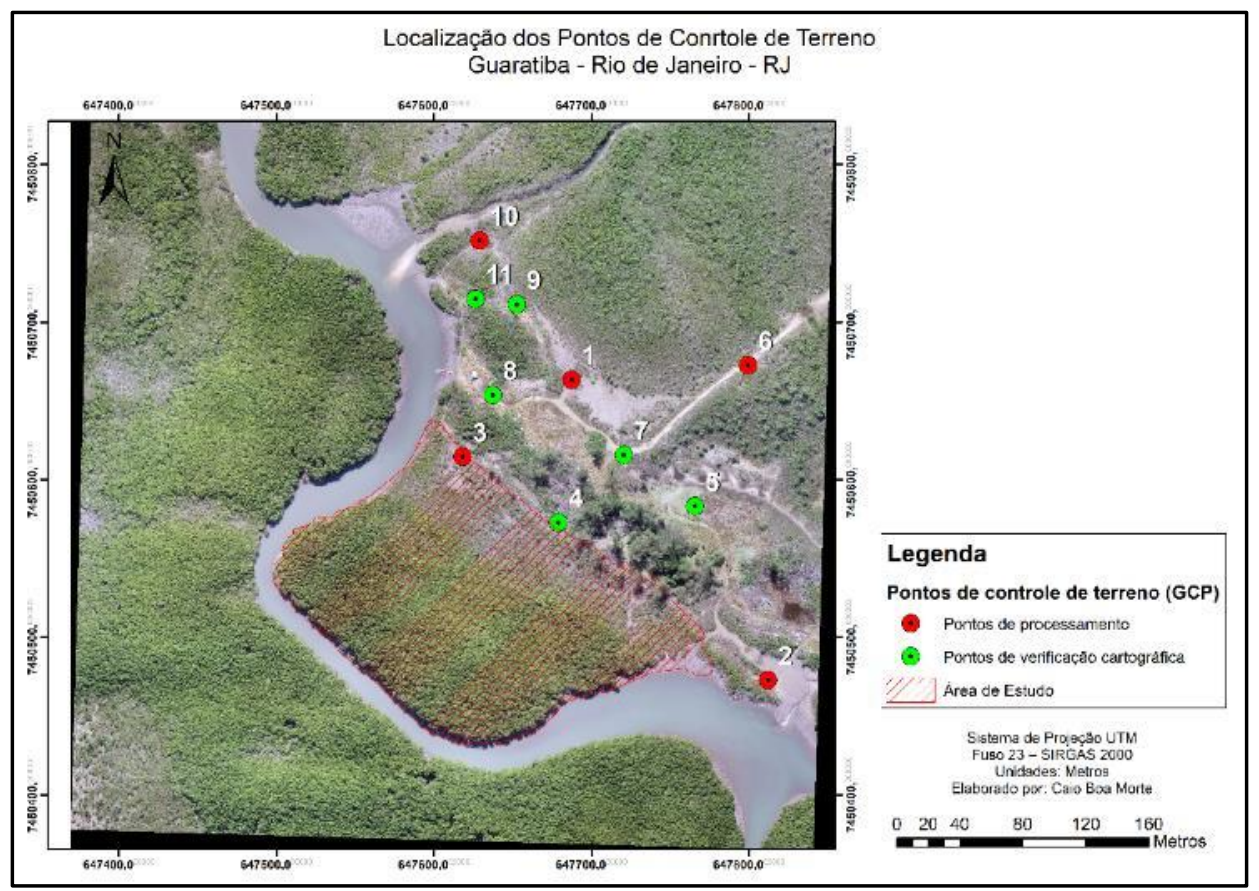

Figura 3: Localização dos pontos para processamento e para avaliação 
Após o processamento no software de fotogrametria, os dados foram analisados através de SIG para extrair coordenadas planimétricas e altimétricas de pontos de solo exposto mais próximos possíveis da vegetação (Figura 4), a fim de obter o maior nível de confiabilidade possível na geração do MDT.

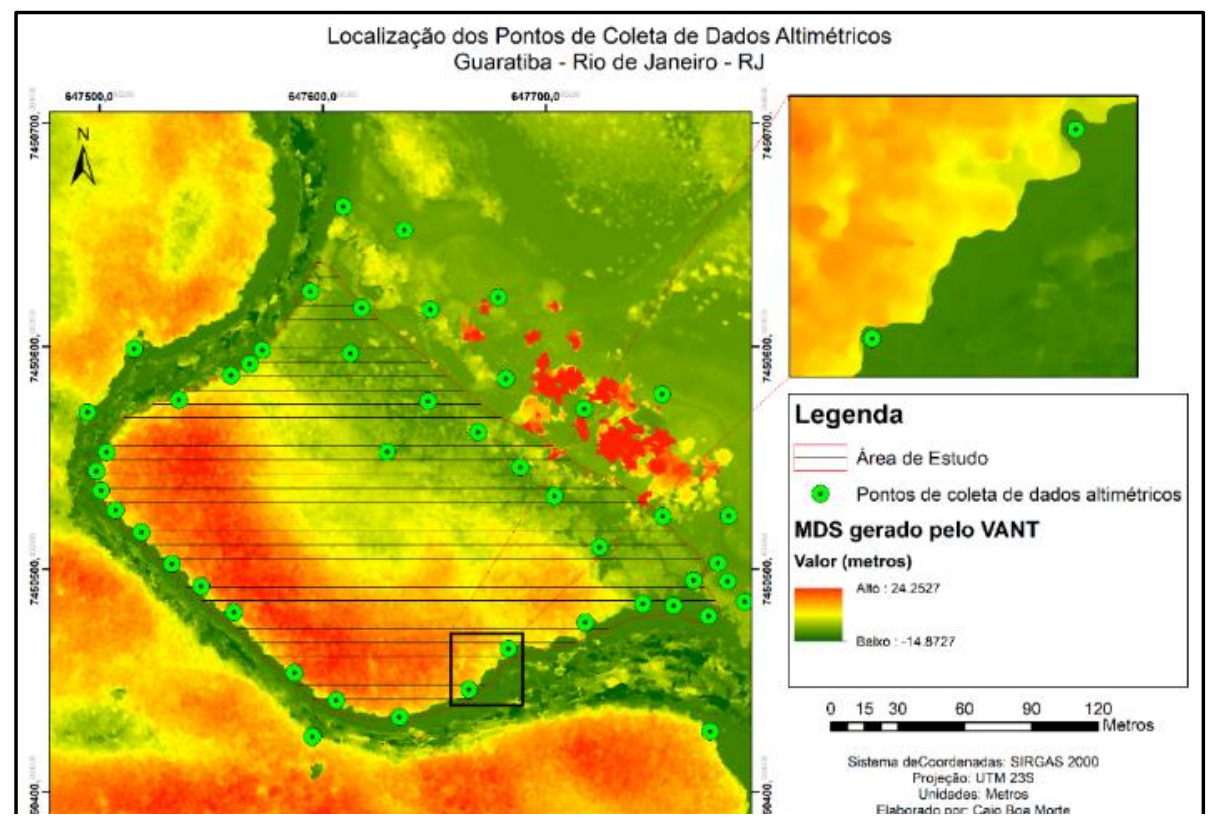

Figura 4: Localização dos pontos de coleta de dados altimétricos

\section{Resultados e Discussões}

Como primeiros resultados temos a produção de duas imagens em raster, o Ortofotomosaico e o Modelo Digital de Superfície (Figura 5). Como explicado por Matese et al. (2016), esses produtos são gerados através de técnicas fotogramétricas de extração de pontos 3D a partir de uma sequência de fotografias aéreas sobrepostas utilizando de algoritmos de Structure from Motion (SfM). Como análise dos primeiros resultados temos uma avaliação expedita dos primeiros produtos gerados na fase do processamento, feita através da análise dos deslocamentos verticais e horizontais entre os valores encontrados no MDS e Ortofoto e os valores determinados através do GNSS de alta precisão (Figura 6). 


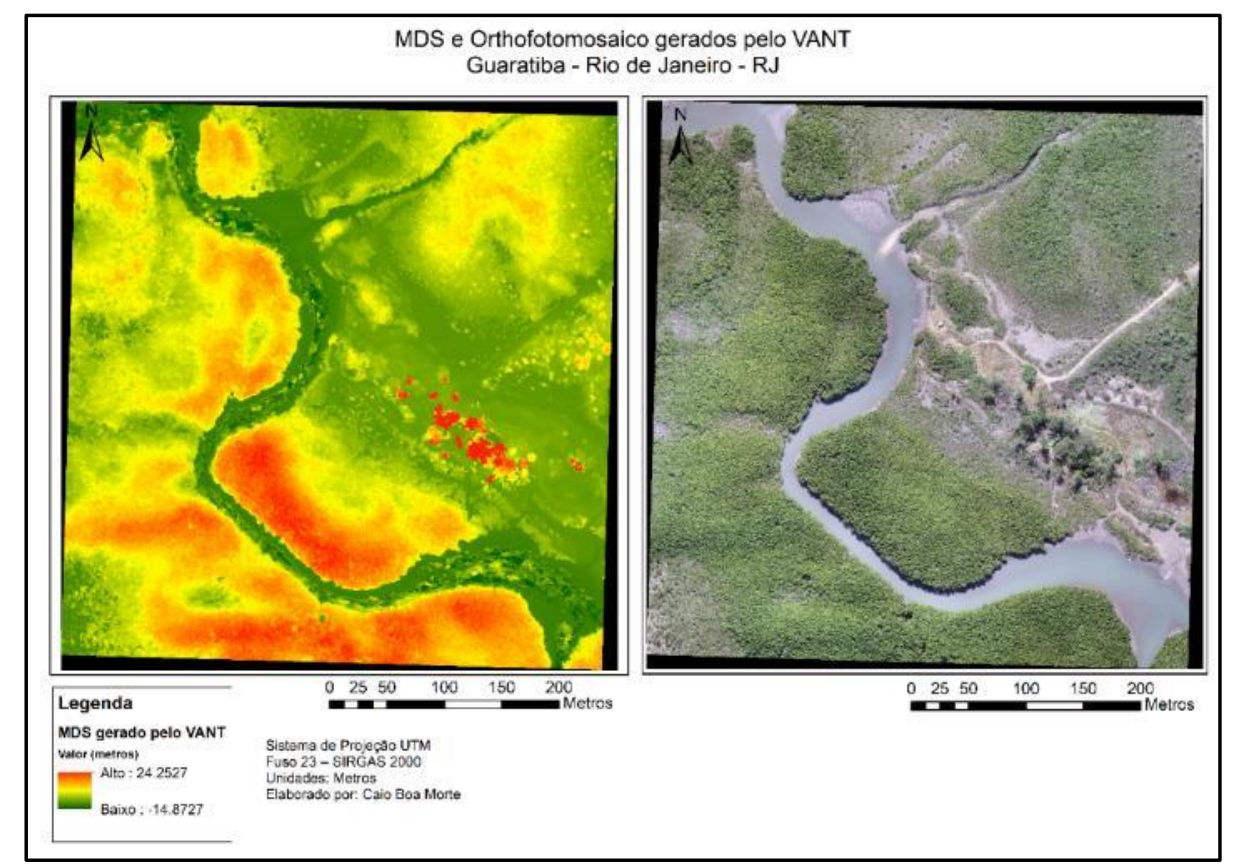

Figura 5: Modelo Digital de Superfície e Ortofotomosaico gerados com dados de VANT

Dito isso, com base nos 6 pontos de avaliação, foi calculado um erro médio de 0,05 metro na altimetria e 0,022 metro na planimetria, com desvio padrão de 0,031 metro na altimetria e 0,011 metro na planimetria. $\mathrm{O}$ erro máximo observado foi de 0,03 metro na planimetria e de 0,10 na altimetria. Constatouse que os dados apresentaram precisão e acurácia compatíveis com o trabalho de Boa Morte (2018), podendo-se concluir que a qualidade dos dados está satisfatória para os propósitos desse estudo.

Utilizando os pontos coletados sobre o MDS como pontos de elevação foi possível realizar uma interpolação dados coletados usando a ferramenta Topo to Raster presente no software Arcgis, a fim de se gerar um Modelo Digital de Terreno (MDT) (Figura 7).

\begin{tabular}{|r|c|c|c|c|}
\hline \multicolumn{5}{|c|}{ Erro $(\mathrm{m})$} \\
\hline \multicolumn{1}{|c|}{ ID } & $\mathrm{X}$ & $\mathrm{Y}$ & $\mathrm{X} \& Y$ & $Z$ \\
\hline 1 & 0.01136 & 0.00234 & 0.01160 & 0.03080 \\
\hline 2 & 0.02688 & 0.00484 & 0.02731 & 0.03331 \\
\hline 3 & 0.00366 & 0.00175 & 0.00405 & 0.07789 \\
\hline 4 & 0.00023 & 0.00291 & 0.00292 & 0.10560 \\
\hline 5 & 0.00956 & 0.00494 & 0.01076 & 0.04942 \\
\hline 6 & 0.00844 & 0.02902 & 0.03022 & 0.01636 \\
\hline 7 & 0.01697 & 0.02308 & 0.02865 & 0.04865 \\
\hline 8 & 0.02691 & 0.00114 & 0.02694 & 0.06502 \\
\hline 9 & 0.02099 & 0.02179 & 0.03025 & 0.09358 \\
\hline 10 & 0.01172 & 0.02834 & 0.03067 & 0.01186 \\
\hline 11 & 0.01531 & 0.02950 & 0.03324 & 0.02113 \\
\hline Máximo & 0.02691 & 0.02950 & 0.03324 & 0.10560 \\
\hline Mínimo & 0.00023 & 0.00114 & 0.00292 & 0.01186 \\
\hline Média & 0.01382 & 0.01360 & 0.02151 & 0.05033 \\
\hline Desv. Pad & 0.008665 & 0.012463 & 0.011627 & 0.03172 \\
\hline
\end{tabular}

Figura 6: Tabela de erros planimétricos e altimétricos do MDS e Orthofotomosaico gerados pelo VANT. Pontos de processamento em vermelho e pontos de avaliação em verde 
Após a validação dos dados foi feita subtração do MDS pelo MDT, gerando assim um Modelo Digital de Copas das Árvores (MDC) (Figura 8), que mostra os valores de altura dos dosséis da vegetação de mangue dessa área.

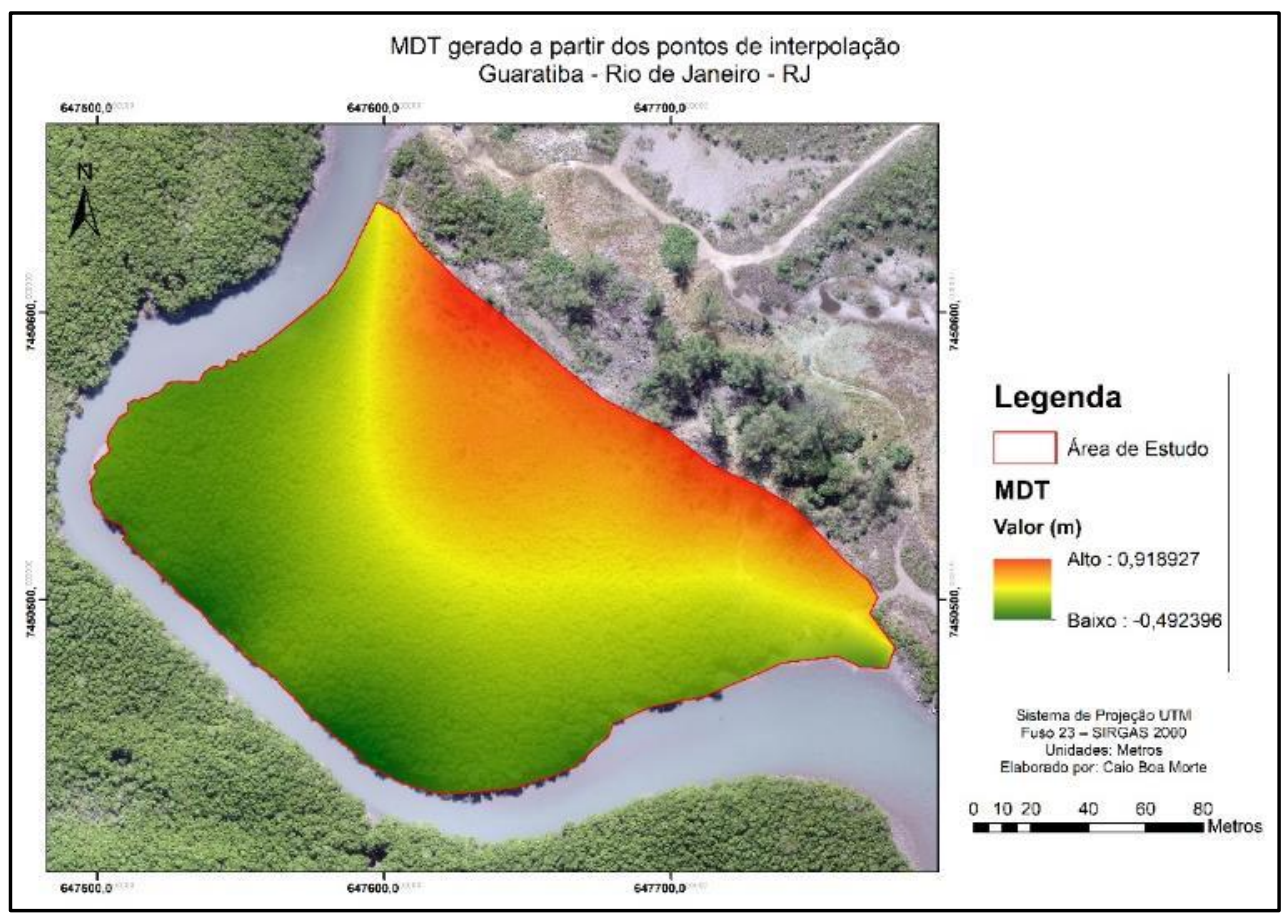

Figura 7: Modelo Digital de Terreno gerado através da interpolação dos pontos altimétricos

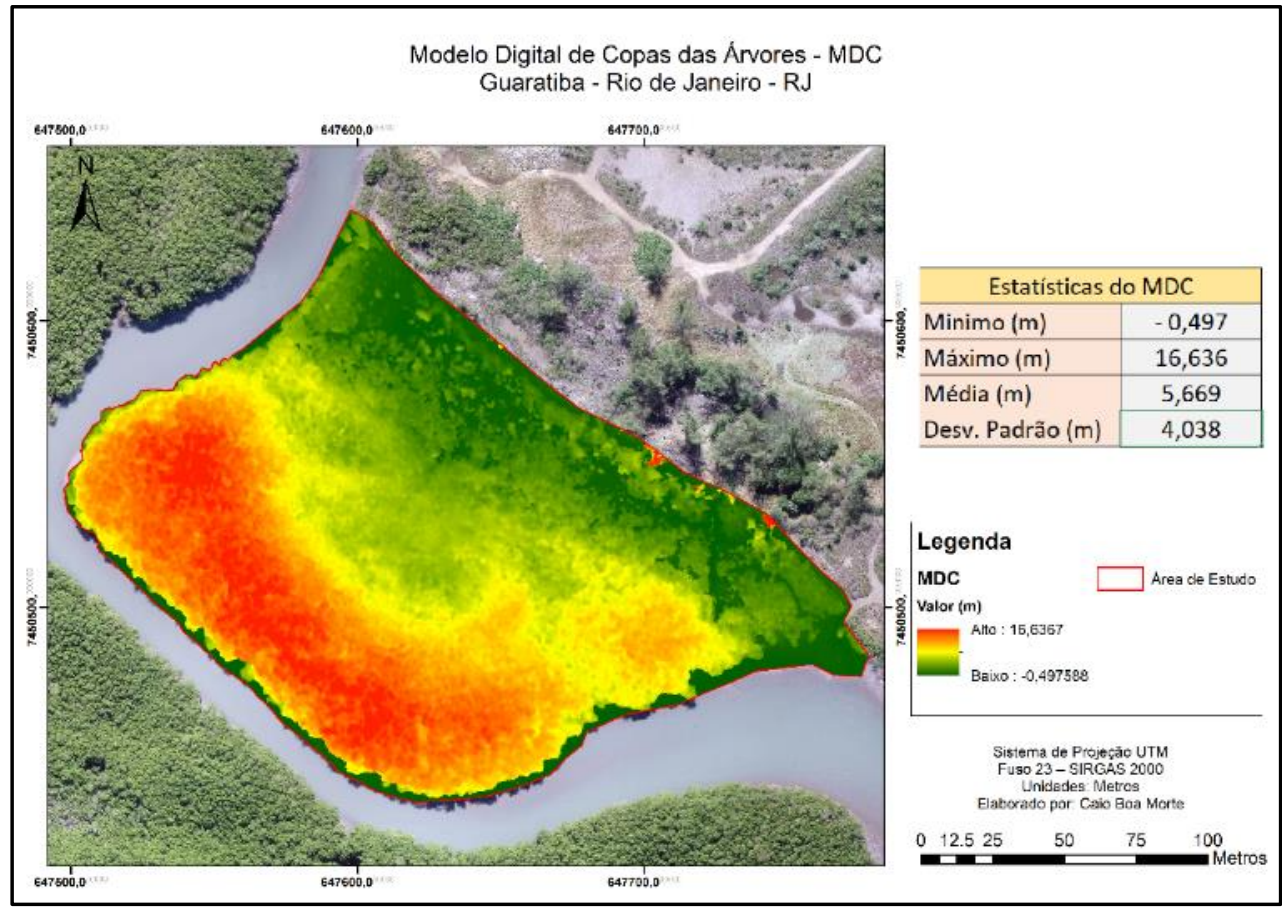

Figura 8: Modelo Digital de Copa das Árvores gerado pela subtração do MDS pelo MDT 
O primeiro ponto a se notar é que mesmo utilizando 48 pontos para geração do MDT são observados valores negativos no MDC. Mesmo que perto de zero, isso ocorre porque em determinados pontos houve uma superestimativa da altitude do terreno. Apesar de serem poucos, esses valores negativos podem acabar impactando no cálculo da altura média da vegetação, já que ele estaria incluindo trechos de solo. Como forma de diminuir esses ruídos, foi feita uma filtragem no MDC eliminando valores inferiores a zero e com isso criando áreas de vazio no MDC, como visto na Figura 9.

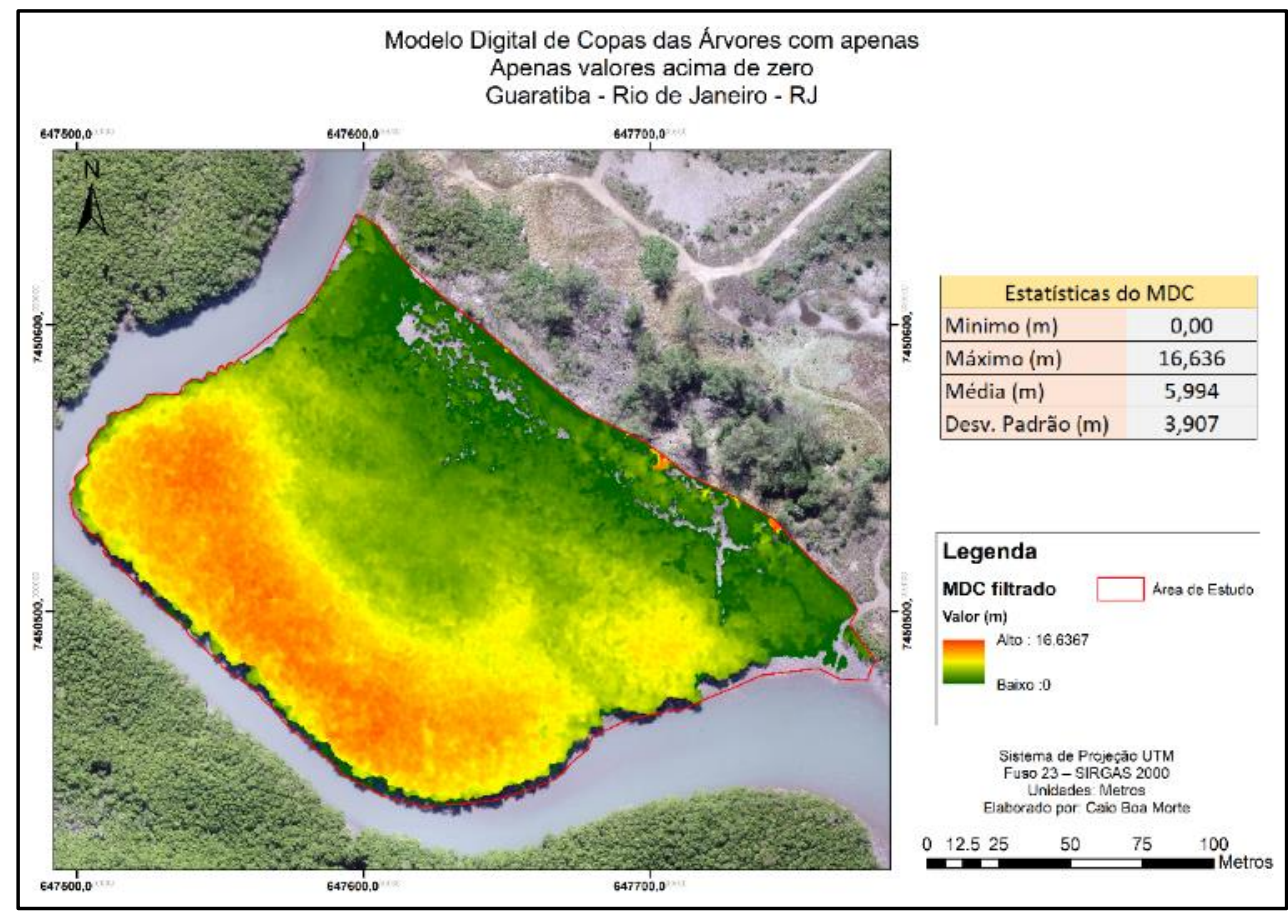

Figura 9: Modelo Digital de Copas das Árvores com valores inferiores a zero removidos

Dito isso, o resultado do MDC não filtrado foi de uma média de altura de dossel de 5, 67 metros, enquanto o resultado do MDC filtrado foi de uma média de 5,99 metros. De acordo com o estudo de Estrada et al. (2014), que teve como área de estudo toda a Reserva Estadual de Guaratiba e que utilizou MDS SRTM para obter a altura da vegetação, a altura média esperada para essa formação é de 7,4 metros com uma margem de erro de 2,3 metros. Ainda que os resultados obtidos sejam compatíveis com Estrada et al. (2014), a margem de erro daquele trabalho é muito maior por causa do insumo utilizado, já que o MDS SRTM costuma apresentar erros aproximados de 10-20 metros, conforme apontam Barros (2006) e Santos (2005).

\section{Conclusões}

Como conclusão podemos afirmar que a utilização de VANT nessa área de estudo com o objetivo de se gerar um MDC de alto detalhamento apresenta indicadores de bons resultados. De acordo com os resultados obtidos neste e nos trabalhos de Boa Morte (2018 e 2019) e Coelho (2019), estima-se que os erros de estimativa de altura das árvores fiquem abaixo de 1 metro, visto que os MDS apresentaram erros máximos de até 15 centímetros e que as variações máximas no terreno - observadas qualitativamente em campo - ficam em torno de 75 centímetros. Em termos médios estes erros devem ficar ainda menores. Esses erros poderiam ser considerados ótimos numa comparação com estimativas 
convencionais em campo, não apenas pelos valores, mas por abranger toda uma área, de forma contínua, e não apenas as estimativas ou medições de alguns indivíduos.

A próxima etapa do trabalho será repetir os levantamentos com medição da altura de alguns indivíduos de mangue mais favoráveis para tal a fim de checar a acurácia dos resultados. É aguardado, ainda, um voo com LIDAR na área para se checar com grande amplitude o MDC, já que este produto oriundo do LIDAR apresenta grande acurácia pois os sinais emitidos pelo equipamento conseguem atingir o solo, gerando um MDT de grande acurácia, como sugere Mejia-Aguilar et al. (2015)

É importante reconhecer que a utilização de VANTs com GNSS RTK embarcados e sensores NIR poderiam trazer informações mais valiosas e detalhadas do que o VANT comercial utilizado nesse estudo. Uma possibilidade seria a utilização do sensor NIR para classificação de diferentes espécies vegetais e, em conjunto com a geração do MDC, calcular com alta precisão o estoque de carbono de diferentes ecossistemas.

\section{Referências Bibliográficas}

BARROS, R. S. Avaliação de Modelos Digitais de Elevação Obtidos a Partir de Sensores Orbitais. Tese de Doutorado. IGEO/UFRJ. Rio de Janeiro. 2006.

BOA MORTE, C. L.; COELHO, R. C. S.; BARROS, R. S. "ANÁLISE DE VEGETAÇ̃̃O ATRAVÉS DE ÍNDICES GERADOS A PARTIR DE DADOS OBTIDOS COM VANT”. 41" Jornada Giulio Massarani de Iniciação Científica, Artística e Cultural da UFRJ, 2019.

BOA MORTE, C. L.; SALVO, D. G. D.; COELHO, R. C. S.; BARROS, R. S. "AVALIAÇÃO DA QUALIDADE DE ORTOFOTOMOSAICO E MDE GERADO A PARTIR DE VANT MULTIROTOR". 40 Jornada Giulio Massarani de Iniciação Científica, Artística e Cultural da UFRJ, 2018.

COELHO, R. C. S. "INVESTIGAÇÃO DE PARÂMETROS DE CORRELAÇÃO ENTRE DADOS FÍSICOS E ESPECTRAIS EM SUBSÍDIO À AVALIAÇÃO DE RESTAURAÇÃO FLORESTAL NA RESERVA BIOLÓGICA DE POÇO DAS ANTAS - RJ: UMA APLICAÇÃO UTILIZANDO VANT”. Dissertação de Mestrado, IGEO/UFRJ.2019.

DONATO, D.C.; KAUFFMAN, J.B.; MURDIYARSO, D.; KURNIANTO, S.; STIDHAM, M.; KANNINEN, M. MANGROVES AMONG THE MOST CARBON-RICH FORESTS IN THE TROPICS. Nat. Geosci. 2011, 4, 293-297.

ESTRADA, G. C. D., SOARES, M. L. G., FERNADEZ, V., \& DE ALMEIDA, P. M. M. (2014). THE ECONOMIC EVALUATION OF CARBON STORAGE AND SEQUESTRATION AS ECOSYSTEM SERVICES OF MANGROVES: A CASE STUDY FROM SOUTHEASTERN BRAZIL. International Journal of Biodiversity Science, Ecosystem Services \& Management, 11(1), 29-35. doi:10.1080/21513732.2014.963676

LAGOMASINO, D., FATOYINBO, T., LEE, S., FELICIANO, E., TRETTIN, C., \& SIMARD, M. (2016). A COMPARISON OF MANGROVE CANOPY HEIGHT USING MULTIPLE INDEPENDENT MEASUREMENTS FROM LAND, AIR, AND SPACE. Remote Sensing, 8(4), 327. doi:10.3390/rs8040327

MATESE, A., DI GENNARO, S. F., \& BERTON, A. (2016). ASSESSMENT OF A CANOPY HEIGHT MODEL (CHM) IN A VINEYARD USING UAV-BASED MULTISPECTRAL IMAGING. International Journal of Remote Sensing, 38(8-10), 2150-2160. doi:10.1080/01431161.2016.1226002 
MEJIA-AGUILAR, Abraham et al., 2015. UAV BASED TREE HEIGHT ESTIMATION IN APPLE ORCHARDS: POTENTIAL OF MULTIPLEAPPROACHES. Bozen, Italy: EGU General Assembly 2015, held 12-17 April, 2015 in Vienna, Austria. id.7082. 2015.

SANTOS, P. R. A. AVALIAÇÃO DA PRECISÃO VERTICAL DOS MODELOS SRTM EM DIFERENTES ESCALAS: UM ESTUDO DE CASO NA AMAZÔNIA. Dissertação de Mestrado. IME. Rio de Janeiro. 2005. 